\title{
Smart Meter Reading System Based on Cognitive Technology
}

\author{
Hu Dongjing, Zu Yunxiao, Hou Bin \\ School of Electronic Engineering, Beijing Univ. of Posts and Telecommunications, Beijing 100876, China \\ \{zuyx \& hdj\}@bupt.edu.cn
}

\begin{abstract}
Smart grid system is an automated power system that monitors and controls grid activities. Smart grid also makes the power network stable, secure, efficient, flexible, economical, and environmental friendly. However, it has constraints in the dedicated frequency resources and poor performance in electricity allocation and communications. As a new developing communication technology, the cognitive radio suggests a way to reuse the limited spectrum resources due to the shortage and low utilization of frequency bands. A new smart meter reading system based on cognitive technology is designed in this paper. In this grid system, smart meter reading system is replaced by an extended wireless sensor network, in which the cognitive radio technology is a key factor for communication. The simulation results explain that the delay, the accuracy and the bit rate of transmitting can satisfy the communication requirements of the grid.

Index Terms - Smart Grid, Meter Reading, Cognitive Radio, Frequency Resources
\end{abstract}

\section{Introduction}

The smart grid is an automated power system that monitors and controls grid activities. Smart grid explores and exploits two-way communication technology, advanced sensing, metering and measurement technology, modern control theory, network grid technology, and machine learning in the power system to make the power network stable, secure, efficient, flexible, economical, and environmental friendly. Novel control technology, information technology, and management technology should be effectively integrated to realize the smart information exchange within the power system, as proposed in [1]. In a word, the goal of smart grid is to systematically optimize the cycle of power generation and utilization.

Recently, a reliable smart grid has been established in China, which communicates mainly using the fiber-optic, microwave, carrier and satellites, with the main feature of hierarchical self-organizing electricity network dedicated communication architecture [2]. However, there are some problems in smart grid, such as the vulnerable backbone architecture which cannot meet the new requirements of the scheduling data network propagation. In addition, the different layers of the communication network cannot achieve the desired integration or full use of resources. Compared to the backbone architecture, the access network is even weaker with low ability to allocate or distribute the electricity in the output power grid. A solution for the communication system is essential to solve the gap between the backbone network and the access network.
In the wireless monitoring and remote meter reading system of the smart grid, the data network should have the following functions: automatic networking, reliable data transmission, and a flexible, low-cost, low-power and secure networking method [3].

Wireless sensor network has recently emerged as a computing platform combined with monitoring, control and communications among a great number of sensor nodes. In this way, if the sensor nodes are added by remote electricity meter reading function in the networks, an available communication solution will be applied between the backbone network and access network.

There is evidence that the dedicated frequency band around $230 \mathrm{MHz}$ is overcrowded, which has restricted the development of the smart grid. What's more, the limited spectrum resources and the lack of the flexibility in spectrum allocation cannot meet the demand of electricity power for the access layer. The cognitive radio (CR) technology enables the secondary user to sense, identify and intelligently access the unoccupied spectrum. Thus the cognitive radio technology provides a viable way to ease the contradiction between the lack of spectrum resources and the growing demand for wireless access. The power will be transmitted through the spectrum holes in the licensed bands by applying the cognitive radio technology in the smart grid. As a result, by increasing the spectral efficiency and data rate, CR will face the need of band switching for more utilization and less interference [4].

This article studies the smart meter reading system based on the cognitive radio technology and focuses on how the cognitive technology is incorporated into the overall smart grid system.

\section{System Model and Structure}

\section{A. System Model}

Smart meter reading system is a cognitive radio system based on the overlay sharing method. The overlay is an access technology with low interference and access interrupt probability. To reduce the interference to the primary user, overlay requires the nodes to work on different frequency bands. In this article, considering the system has $M$ primary users (PU) and $N$ secondary users (SU), and the primary users work in the dedicated frequency band around $230 \mathrm{MHz}$ or the ZigBee band 2.4GHz, as shown in Fig.1 [5]. 


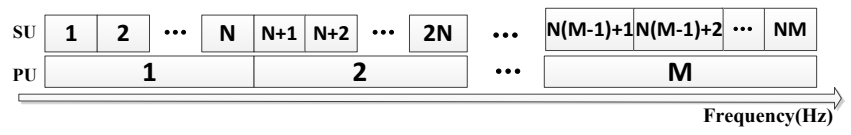

Fig.1 Channels for a Cognitive Model

Once is allocated a sharing spectrum, the secondary users can use the adaptive modulation in this present band to improve the transmission performance. The secondary users can only communicate with the primary users. Thus the primary users, acting as central node (also known as aggregation node), determine which frequency band to be selected and then tell the corresponding secondary user. However, the secondary user does not switch the information to other secondary users.

In the scenario of a building, the sensor nodes at a story collect the power data and send it to the aggregation node regularly. The sensor nodes can sense the unused bands and channels, and send the collecting information to the aggregation node. Then the aggregation node collects all the sensing information from multiple sensor nodes, it will find a channel suitable for power data transmission by applying a selecting strategy. Finally, the aggregation node will give a reply message to the corresponding sensor node. The message contains the sub-channel identification in FDMA or the time slot flag in TDMA to avoid the interference among the nodes or the channel overlap. Because of the different utilization of the licensed bands for the primary users at different times, the system may choose a different band to transmit data. However, in order to make full use of the spectrum resources, the channel at the same story may use the same frequency band. As an exception, the nodes in the same story may use more than one channel temporarily when one channel cannot support the communication itself, such as in the spectrum switching process. But nodes at neighbor stories have to use different frequency band to avoid the interference. The licensed frequency bands at present are shown in Table I [6].

TABLE I Global Spectrum Allocation

\begin{tabular}{|l|l|}
\hline Broadcast Band & $535-108 \mathrm{kHz}$ \\
\hline TV signals & $54-890 \mathrm{MHz}$ \\
\hline & $824-849 \mathrm{MHz}$, \\
GSM cellular phones & $880-915 \mathrm{MHz}$, \\
& $925-960 \mathrm{MHz}$ \\
\hline \multirow{2}{*}{ Global Positioning System } & $1575.42 \mathrm{MHz}$, \\
& $1227.60 \mathrm{MHz}$ \\
\hline Microwave Oven & $2.45 \mathrm{GHz}$ \\
\hline Live System & $11.7-12.5 \mathrm{GHz}$ \\
\hline Ultra-wideband radio & $3.1-10.6 \mathrm{MHz}$ \\
\hline
\end{tabular}

In this paper, the ZigBee frequency band and dedicated band $230 \mathrm{MHz}$ for grid are used for data transmission. The ZigBee protocol works at the band of $2.4 \mathrm{GHz}$ and $868 / 915 \mathrm{MHz}$ for the physical layer. The ZigBee has advantages of low cost, low power consumption, great network capacity, short delay, high reliability and security. It can also meet the demand of the grid data transmission because the data rate for smart grid is measured in bits [7]. As a result, the system provides the frequency bands of dedicated grid $230 \mathrm{MHz}$ and ZigBee $2.4 \mathrm{GHz}$ or $868 / 915 \mathrm{MHz}$ physical channels to be chosen, and introduces the cognitive radio technology to reuse the current available idle channel for data transmitting.

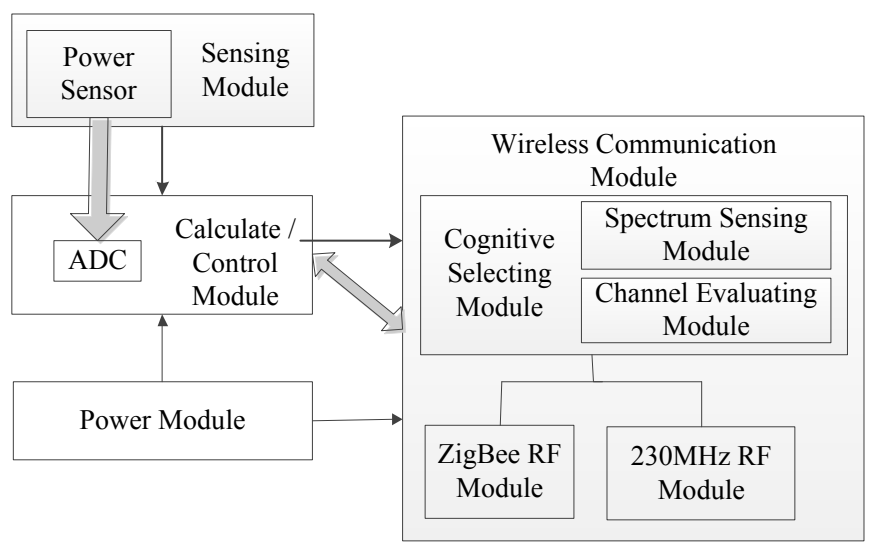

Fig. 2 Structure of a Sensor Node

\section{B. Structure of the Nodes}

The structure of nodes in the smart meter reading system is similar to those in wireless sensor network, as shown in Fig.2. The node in smart meter reading system composes of four parts--the sensor module, the calculating and control module, the wireless communication module and the power module. The wireless communication module comprises ZigBee $/ 230 \mathrm{MHz}$ frequency module and cognitive selecting control module. In addition, the sensor module is designed separately and connects to the control module by the extended interface for a faster updating and replacement during the monitoring assignment [8-9].

To meet the power demand of a building, it is essential to design a self-organizing and low-power data collecting scheme based on the cognitive radio techniques for wireless sensor networks. The power data in buildings, which is measured by bits, need a relatively narrow band to be transmitted. Despite the fact that the sensor node can just provide low power for collecting data at a low bit rate, the bandwidth of the wireless sensor network is still qualified for the smart grid. Therefore, aimed at a simple and efficient data collecting strategy for practical use, the system in this article is established on IEEE 802.15.4/ZigBee protocol and the power model for a dedicated frequency band at $230 \mathrm{MHz}$. The deployment of the sensor nodes is shown in Fig.3.

A different number of sensor nodes are placed in rooms, depending on the size of the room and the required accuracy of the monitoring. The sensor nodes form a network by selforganizing. During the deployment, operators have to guarantee the communication quality and balance the energy consumption according to the signal strength and the power value of the central node and its adjacent nodes. Considering the limited resources of the sensor nodes the interaction of 
control information among the sensor nodes cannot be complex or too much. It is also very important to keep equilibrium of the consumption among all the nodes in the network, which keeps the network running for a longer time.

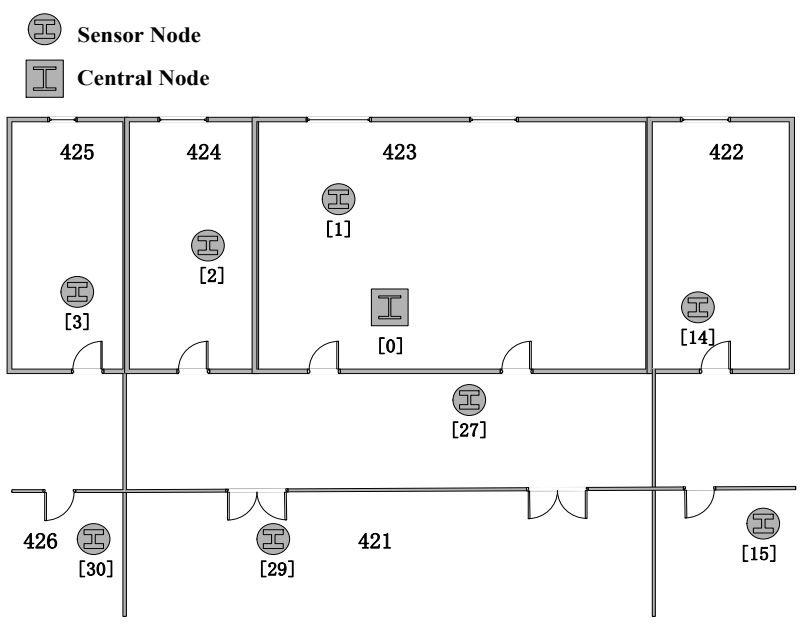

Fig. 3 Nodes Deployment on the Floor

\section{Simulation and Analysis}

\section{A. Simulation Scheme}

In order to verify the feasibility of applying the cognitive radio to the smart meter reading system the simulation is done. The simulation executes as followings. When the smart meter reading system has power data to transmit, the transmitter will inspect all the channels to select a channel most suitable for the power data transmitting [10]. Once selected, the channel will be accessed and starts to transmit data. At the same time, the selected channel has to be inspected at regular intervals to guarantee a reliable and secure data transmission until the simulation is finished. If the channel remains idle and available for the data transmitting, the transmission will continue; if not, the transmitter has to go back to the inspecting step again. The inspecting step means that the transmitter inspects and selects an available channel for data transmission regularly.

Taking 3 channels as example in this simulation, and the three channels are sorted in accordance with the channel quality. It means that the channel marked No.1 is the most suitable for transmitting power signals, which matches the features of the signal best. Then the channel marked No.2 is not as suitable as No.1 channel, and No.3 channel is the worst among the three channels. The signal is modulated in 2FSK mode. During the transmission, the transmitter will check the occupancy of the channel periodically to determine whether to change into another channel when occupied. The switching process of the channels is shown in Fig.4. A delay will exist if some of the packets get lost and have to be retransmitted during the channel switching due to the Automatic Repeat Request (ARQ) function.

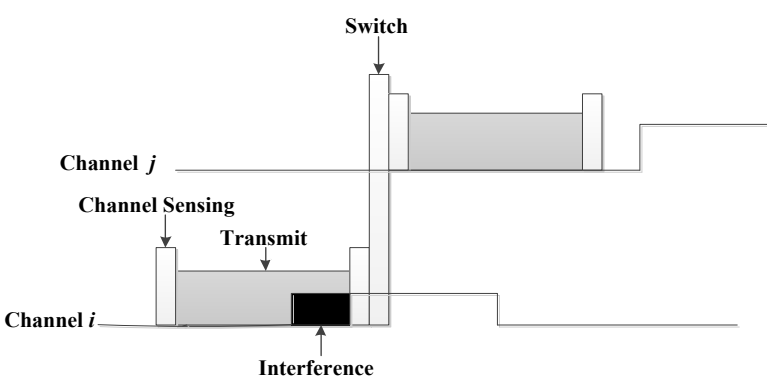

Fig. 4 Switching of the System

\section{B. Performance Analysis}

The simulation system has 100 packets to transmit on the 3 channels, where a packet contains 500 bits. The working channel has to be inspected at a regular interval of sending 9 packets. According to the inspecting results, the transmitter will continue to send signals over the channel if the channel is still available; if not, the transmitter has to inspect all the channels again to find an unused one to transmit packets. Assuming that at the beginning of simulation channel No.1 is unused and available for data to transmit, and channel No. 2 and channel No. 3 are occupied at the same time. It is obvious that the packets will be transmitted over channel No. 1, as indicated in the simulation process. After transmitting 39 packets, the channel No.1 is occupied and its quality turns bad. However, the transmitter won't get aware of the occupied channel until the next regular inspecting time. The channel No.2 is inspected becoming idle but channel No.3 remains occupied at the moment. Therefore the system switches onto channel No.2 to continue transmitting. As the last few packets are unable to be identified at the receiver when channel No.1 becomes bad, the lost data packets have to be transmitted again via channel No.2. What's more, delay occurs in the switching process between the two channels. The parameters of the simulation results are shown in Table II, in which 1 slot means the length of time required to transmit a packet.

Fig.5 indicates the occupancy results of 3 channels through the simulation. All the 3 channels are ordered from best to worst depending on the match degree of channel condition and transmission request. Channel No.1 is idle at first and data is transmitted over it. From the 40th data packet, channel No.1 comes bad due to the occupation of the primary users or interference. The packet loss does not occur at first until channel No.1 is bad enough. Packet loss will continue until it is inspected that channel No.1 is under the qualified threshold. Then data is switched to channel No.2 to transmit.

TABLE II Parameters of the Simulation System

\begin{tabular}{|l|l|}
\hline Channel inspection period & 9 slots \\
\hline Data packets to transmit & 100 \\
\hline Size of a packet & $500 \mathrm{bit}$ \\
\hline Bit rate & $1000 \mathrm{bit} / \mathrm{s}$ \\
\hline Centre frequency & $1000 \mathrm{~Hz}$ \\
\hline Minimum threshold of signal-to-noise ratio & $-5 \mathrm{~dB}$ \\
\hline Number of channels & 3 channels \\
\hline
\end{tabular}



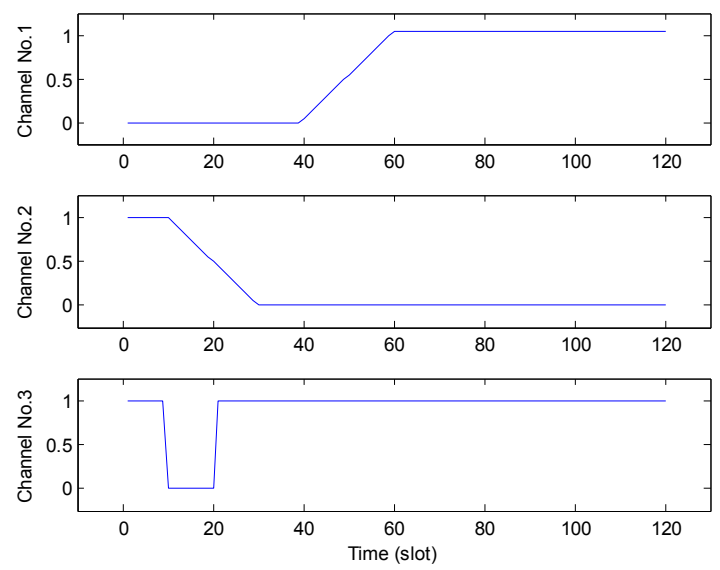

Fig. 5 Occupancy of the three channels

Table III depicts the simulation results. Originally there are 100 data packets to be transmitted but 110 packets are sent in fact. The extra 10 packets are for the retransmission of the lost packets during switching process, which can be calculated by the transmitted packets minus the sent packets. It reveals that the switch delay almost equals the time for transmitting 10 packets. But the delay and the retransmission don't have a bad effect on receiving or calculating the packets in the central terminal. So we draw a conclusion that as to the smart grid meter reading system, the utilization of the cognitive radio technology in wireless data transmission can effectively guarantee the security and reliability.

TABLE III Data of Simulation Results

\begin{tabular}{|l|l|}
\hline Number of data packets prepared to transmit & 100 \\
\hline Number of packets sent over real channels & 110 \\
\hline Number of received packets & 100 \\
\hline Packet loss & 10 \\
\hline
\end{tabular}

\section{Conclusions}

This paper studies how to apply the cognitive radio technology to the smart meter reading system, so as to resolve the tension of the spectrum resources near the $230 \mathrm{MHz}$ frequency band and the lack of flexibility of the spectrum allocation. The scheme is aimed at meeting the demand of the access layer in smart grid. Then accordingly, a new grid system based on cognitive radio technology is designed, where the traditional meter reading terminal is replaced by wireless sensor node with meter reading function, and the communication is done by the means of cognitive technology. The feasibility of the scheme has been verified in simulation.

\section{Acknowledgment}

This research is supported by the State Key Lab. of Power System, Dept. of Electrical Engineering, Tsinghua University, Beijing 100084, China.

\section{References}

[1] S. Massoud Amin and B.F. Wollenberg, "Toward a smart grid: power delivery for the 21st century[C]," IEEE Power and Energy Magazine. Sep.-Oct. 2005, pp. 34-41.

[2] Yan Zhang, "Dynamic Spectrum Access in Cognitive Radio Wireless Networks[C]," IEEE International Conference on Communications. Beijing, 2008, pp. 4927-4932.

[3] A.A. Sreesha, S. Somal, and Lui Tai, "Cognitive Radio Based Wireless Sensor Network architecture for smart grid utility[C]," Systems, Applications and Technology Conference, IEEE Long Island, 2011, pp. 1-7.

[4] Qiu R C, Zhen Hu, and Chen Zhe, et al, "Cognitive Radio Network for the Smart Grid: Experimental System Architecture, Control Algorithms, Security, and Microgrid Testbed[C]," IEEE Transactions on Smart Grid, 2011, pp. 724-740

[5] G. Cheng, W. Liu, Y.Z, and Li, W.Q. Cheng, "Spectrum Aware Ondemand Routing in Cognitive Radio Networks[C]," 2nd IEEE International Symposium on New Frontiers in Dynamic Spectrum Access Networks (DySPAN) 2007, pp. 571-574.

[6] D.M. Pozar, Microwave Engineering (3rd Edition), Beijing: Publishing House of Electronics Industry, July 2009, pp. 1-10.

[7] K.L. Alcin Yau, P. Komisarczuk, and Paul D. Teal. "Cognitive Radiobased Wireless Sensor Networks: Conceptual Design and Open Issues," 2nd IEEE Workshop in Wireless and Internet Services (WISe) 2009. New Zealand, Oct.2009, pp. 955-962.

[8] H.L. Smith, "DA/DSM Directions-An Overview of Distribution Automation and Demand Side Management with Implications of Future Trends [J]," IEEE Computer Application in Power, Oct.1994, pp. 122128.

[9] J.J. Cárdenas, L. Romeral, and A. Garcia, "Load forecasting framework of electricity consumptions for an Intelligent Energy Management System in the user-side," Expert Systems with Applications, Vol. 39, No. 5, April 2012, pp. 5557-5565.

[10] I.F. Akyildiz, W. Lee, and M.C. V.S. Mohanty, "Next Generation/ Dynamic Spectrum Access/ Cognitive Radio Wireless Networks, A Survey [J]," Computer Networks, 2006. 